\title{
Fiber Type Composition and Maximum Shortening Velocity of Muscles Crossing the Human Shoulder
}

\author{
R.C. SRINIVASAN, ${ }^{1}$ M.P. LUNGREN, ${ }^{1}$ J.E. LANGENDERFER, ${ }^{2}$ AND R.E. HUGHES ${ }^{2,3 *}$ \\ ${ }^{1}$ Medsport and Orthopaedic Research Laboratories, Medical School, University of Michigan, Ann Arbor, Michigan \\ ${ }^{2}$ Department of Biomedical Engineering, University of Michigan, Ann Arbor, Michigan \\ ${ }^{3}$ Department of Orthopaedic Surgery, University of Michigan, Ann Arbor, Michigan
}

\begin{abstract}
A study of the fiber type composition of fourteen muscles spanning the human glenohumeral joint was carried out with the purpose of determining the contribution of fiber types to overall muscle cross-sectional area (CSA) and to estimate the maximum shortening velocity $\left(V_{\max }\right)$ of those muscles. Muscle biopsies were procured from 4 male cadavers (mean age 50) within $24 \mathrm{hr}$ of death, snap frozen, mounted, and transversely sectioned $(10 \mu \mathrm{m})$. Slides were stained for myofibrillar ATPase after alkaline preincubation. Photoimages were taken of defined areas (100 fibers) using the Bioquant system, and fiber type and CSA were measured from these images. Staining for mATPase produced three different fiber types: slow-oxidative (SO), fast-oxidative-glycolytic (FOG), and fast-glycolytic (FG). On average, the muscle fiber type composition ranged from 22 to $40 \%$ of FG, from 17 to $51 \%$ of FOG, and from 23 to $56 \%$ of SO. Twelve out of the 14 muscles had average SO proportions ranging from 35 to $50 \%$. $V_{\max }$ was calculated from the fiber type contribution relative to CSA and shortening velocity values taken from the literature. The maximum velocities of shortening presented here provide a physiological basis for the development of human shoulder musculoskeletal models suitable for predicting muscle forces for functionally relevant tasks encompassing conditions of muscle shortening and lengthening. Clin. Anat. 20:144-149, 2007. @2006 Wiley-Liss, Inc.
\end{abstract}

Key words: shoulder; elbow; muscle architecture; fiber type

\section{INTRODUCTION}

Maximum velocity of muscle shortening is an important parameter in musculoskeletal models (McGill and Norman, 1986; Kaufman et al., 1991; Thelen, 2003). In formulations accounting for force generation during eccentric or concentric muscle contractions, a muscle's shortening velocity at a point in time is normalized against its maximum shortening velocity to determine muscle force (Hatze, 1978, 1981; Zajac, 1989). It has been shown that muscles are able to effectively generate muscle force under shortening or lengthening contractions in proportion to the fiber type composition (Bárány, 1967; Thorstensson et al., 1976). Fiber type composition can be determined by histochemical staining used to identify the mATPase activity according to three groups: type I, type IIa, and type IIb (Burke, 1981). More recently immunohistochemical assays have been used to correctly identify previously considered type IIb fibers as type IIx (Smerdu et al., 1994; Bamman et al., 1998; Scott et al., 2001). In their review article detailing fiber type staining methodologies, Scott et al. (2001) stated that the I, IIa, and IIx fiber types can be identified by their relative speed and oxidative/glycolytic capabilities as slow-oxidative (SO),

*Correspondence to: R.E. Hughes, Ph.D, MedSport, University of Michigan, 24 Frank Lloyd Wright Drive, POB 391, Ann Arbor, Michigan 48106-0391. E-mail: rehughes@umich.edu

Grant sponsor: Whitaker Foundation; Grant number: TF-03-006.

Received 6 May 2005; Revised 8 January 2006; Accepted 16 February 2006

Published online 22 June 2006 in Wiley InterScience (www. interscience.wiley.com). DOI 10.1002/ca.20349 
TABLE 1. Sampling of Fibers for Analysis in Shoulder Muscles

\begin{tabular}{lcccr}
\hline Muscle & Abbrev. & $N$ (specimens) & $N$ (areas) & $N$ (fibers) \\
\hline Anterior deltoid & AD & 4 & 22 & 1,603 \\
Middle deltoid & MD & 4 & 31 & 2,124 \\
Posterior deltoid & PD & 4 & 35 & 2,036 \\
Coracobrachialis & Cb & 1 & 9 & 898 \\
Infraspinatus & Inf & 3 & 13 & 768 \\
Subscapularis & SbS & 4 & 29 & 2,033 \\
Supraspinatus & SSP & 3 & 20 & 1,119 \\
Teres minor & Tmi & 2 & 7 & 495 \\
Teres major & Tma & 4 & 30 & 1,579 \\
Latissimus dorsi & LD & 3 & 45 & 1,896 \\
Long head biceps & LHB & 3 & 22 & 1,832 \\
Short head biceps & SHB & 3 & 24 & 1,599 \\
Long head triceps & Tri & 4 & 41 & 3,013 \\
Pectoralis & Pec & 3 & 31 & 1,580 \\
\hline
\end{tabular}

fast-oxidative-glycolytic (FOG) and fast-glycolytic (FG), respectively. Consequently, the force generating potential of muscle under shortening or lengthening conditions is dependent on the relative contributions of muscle fibers containing the various myosin isoforms, slow-oxidative (SO), fast-oxidative-glycolytic (FOG), and fast-glycolytic (FG) fibers, to the muscle as a whole. As their names suggest, each fiber type is known to possess different maximum shortening velocities, which are described in some range (Thorstensson et al., 1976; Larsson and Moss, 1993; Larsson et al., 1997; Cheng et al., 2000) for each muscle fiber type.

Previous studies have examined the fiber type composition for a subset of upper extremity muscles (Jennekens et al., 1971; Johnson et al., 1973) or for individual upper extremity muscle (Jozsa et al., 1978; Fitts et al., 1989; Klitgaard et al., 1990; Jürimäe et al., 1996; Trappe et al., 2000); in addition, fiber type cross-sectional areas (CSA) have been measured in supraspinatus and deltoid muscles (Gansen and Irlenbusch, 2002). However, a complete set of fiber typing and CSA analysis data for all of the 14 muscles that cross the human glenohumeral joint is unavailable.

There were three objectives of this study: (1) to measure the relative fiber type composition of 14 muscles spanning the glenohumeral joint in muscle biopsies from recently deceased human bone and soft tissue donors; (2) to determine the contribution of each fiber type to the overall muscle CSA; and (3) to estimate the maximum shortening velocity of each of these muscles.

\section{MATERIALS AND METHODS}

Up to 14 muscles crossing the glenohumeral joint were dissected from four adult human males within $24 \mathrm{hr}$ of death. All muscle biopsies were obtained from anatomical donors intended for bone and soft tissue recovery for transplant; that is, the dissections and muscle biopsies performed for this study were secondary to bone recovery for transplant. The tissue recovery process, specifically the removal of the humerus, led to the disruption of the muscles requested for this study. As a result, complete sets of all 14 muscles were not obtained from each donor. Additionally, volumes of muscle were not consistently obtainable from specific different depths of the same muscle. Thus, volumes obtained were classified only by muscle region (anterior deltoid vs. middle deltoid, etc.). The donors ranged in age from 17 to 75 years-old (mean 50); each donor was screened by the tissue procurement agency for research suitability and had no history of shoulder surgery, musculoskeletal injury, or musculoskeletal disease.

Muscle samples were removed from the right side of the body, snap frozen at $-196^{\circ} \mathrm{C}$ in liquid nitrogen, and stored at $-80^{\circ} \mathrm{C}$. The muscle samples were later divided into blocks approximating $1 \mathrm{~cm}^{3}$ and mounted in Optimal Cutting Temperature embedding medium (Sakura Finetek, Torrance, CA). After warming the blocks to $-25^{\circ} \mathrm{C}$, transverse sections $(10 \mu \mathrm{m})$ were cut using a cryostat. Sections (6-10 per block) were mounted on gelatin coated slides and air dried for between $15 \mathrm{~min}$ and $1 \mathrm{hr}$ prior to staining. Following alkaline preincubation at a $\mathrm{pH}$ of 10.4 , the sections were stained for myofibrillar ATPase at a pH of 9.4, according to the procedure described by Guth and Samaha (1970) with the same modifications made by Singh et al. (2002).

A pilot study was conducted to assess the inter-rater reliability of fiber typing and CSA determination, as described by Portney and Watkins (1993). Two people independently graded the type and the CSAs of 100 fibers.

The average fiber type proportion was determined across the four donors. As described by Singh et al. (2002), areas (each of $\sim 50-100$ fibers) were selected from a muscle section for analysis. Areas were chosen based on the following exclusion criteria: two toned fibers, lack of distinct fiber outline, and incomplete muscle fibers. The specific number of areas and muscle of origin are listed in Table 1. Photoimages of each of the areas were taken using the Bioquant system with a camera (BIOQUANT Image Analysis Corporation, Nashville, TN) connected to a light microscope. The fiber type classification was carried out in Photoshop 7.0 (Adobe Systems, San Jose, CA) by the two individuals who took part in the inter-rater reliability pilot study. According to the criteria outlined by McIntosh et al. (1985) and Richmond et al. (1999) lightly staining fibers were classified as type I or slow-oxidative (SO), intermediate staining fibers were classified as type IIa or fast-oxidative-glycolytic (FOG), and dark staining fibers were classified as type IIx or fast-glycolytic (FG). The muscle of origin and the corresponding number of specimens, areas, and fibers are listed in Table 1. Average fiber type proportions were calculated for each muscle and recorded in Table 2.

Fiber CSA was also measured for each of the three fiber types using Photoshop 7.0. According to the procedure described by Singh et al. (2002), 20 fibers of each type 
TABLE 2. Relative Proportions of Fiber Types, Contributions of Each Fiber Type to Whole Muscle CSA, and the $\boldsymbol{V}_{\max }$ Values for Shoulder Muscles

\begin{tabular}{|c|c|c|c|c|c|c|c|}
\hline & \multicolumn{3}{|c|}{ Mean \% (SD) } & \multicolumn{3}{|c|}{ CSA \% } & \multirow{2}{*}{$\begin{array}{c}V \max \\
(\mathrm{Lo} / \mathrm{s})\end{array}$} \\
\hline & FG & FOG & SO & FG & FOG & SO & \\
\hline$A D$ & $27(11)$ & $25(10)$ & $47(9)$ & 21 & 23 & 55 & 1.2 \\
\hline MD & $30(10)$ & $23(9)$ & $47(12)$ & 33 & 21 & 46 & 1.6 \\
\hline PD & $27(13)$ & 17 (6) & $56(14)$ & 28 & 14 & 57 & 1.4 \\
\hline $\mathrm{Cb}$ & 26 & 51 & 23 & 17 & 54 & 29 & 1.3 \\
\hline Inf & $29(10)$ & $23(11)$ & $48(14)$ & 23 & 24 & 54 & 1.3 \\
\hline SbS & $38(9)$ & $25(7)$ & 37 (10) & 34 & 24 & 42 & 1.6 \\
\hline SSP & $29(14)$ & $21(5)$ & $50(15)$ & 28 & 21 & 51 & 1.4 \\
\hline Tmi & $22(7)$ & $29(8)$ & $49(11)$ & 18 & 29 & 53 & 1.2 \\
\hline Tma & $23(10)$ & 29 (11) & $48(14)$ & 20 & 32 & 48 & 1.2 \\
\hline LD & $35(9)$ & 17 (10) & $48(12)$ & 33 & 20 & 47 & 1.6 \\
\hline LHB & 36 (15) & $25(10)$ & 39 (10) & 37 & 31 & 32 & 1.8 \\
\hline $\mathrm{SHB}$ & $40(13)$ & $23(13)$ & $37(8)$ & 35 & 26 & 38 & 1.7 \\
\hline Tri & $30(11)$ & $27(14)$ & $44(14)$ & 29 & 28 & 43 & 1.5 \\
\hline Pec & $40(14)$ & $25(14)$ & $35(7)$ & 38 & 26 & 36 & 1.8 \\
\hline
\end{tabular}

were selected and analyzed from three representative areas in up to fourteen muscles from each of the four donors. Average CSA ratios were calculated for the slowoxidative and/or fast-oxidative-glycolytic fibers for each individual muscle. These ratios were used to calculate the relative contribution to total muscle CSA by each fiber type (Table 2).

Maximum velocities of shortening were calculated by weighting the velocities of shortening for each fiber type by the relative contribution to total muscle CSA. Velocities of shortening for slow-oxidative $(0.35 \mathrm{~L} / \mathrm{sec})$, fast-oxidativeglycolytic (1.07), and fast-glycolytic (3.68) fibers were taken from the literature of Larsson and Moss, (1993). Two-sample $t$-tests were used to test for differences in mean fiber types between agonists and antagonists for shoulder rotation, ab/adduction, flexion/extension, and elbow flexion/extension.

\section{RESULTS}

The results of the pilot study determined intraclass correlation coefficients of 0.99 for both determination of fiber type, and determination of fiber CSA. Our method for determining these measures was therefore determined to be reliable. For each muscle the number of specimens and areas sampled, as well as the total number of fibers analyzed, were recorded (Table 1). Across the four donors over 22,000 muscle fibers were analyzed. The mean and range of fiber type proportions for the muscles varied considerably (Table 2 ). On average, the muscle fiber type composition ranged from 22 to $40 \%$ of $F G$, from 17 to $51 \%$ of $F O G$, and from 23 to $56 \%$ of SO. For all muscles except coracobrachialis, pectoralis, subscapularis, and biceps (short head), So type fibers were generally more highly present ( 37 to $56 \%$ ) than the other types.

The relationship between the different fiber types was observed by plotting the percentages of FG or FOG fiber types against the slow fiber types (SO) for each of the muscles measured (Fig. 1). The percentages of FOG and SO fibers were inversely correlated with each other $\left(r^{2}=0.42\right.$, $P<0.05)$, and the proportions of FOG and FG $\left(r^{2}=0.10\right)$ and FG and SO $\left(r^{2}=0.22\right)$ were not correlated with one another $(P>0.05)$.
Most of the studied muscles (12/14) had average SO proportions of $35-50 \%$. Two muscles did not fall in this range. The posterior deltoid had a particularly high average SO proportion of $56 \%$, whereas the coracobrachialis had a low average SO proportion of $23 \%$. Coracobrachialis was the only muscle with FOG fibers, making the largest contribution (51\%). However, these findings may not be typical as samples of coracobrachialis were obtained from only one specimen.

We tested for differences in the relative proportions of the different fiber types between elbow flexors and extensors and found no significant differences. We grouped muscles according to their function at the shoulder (flexors vs. extensors, abductors vs. adductors, internal vs. external rotators), and found no statistically significant differ-

\section{Relative Fiber-Type Percentages}

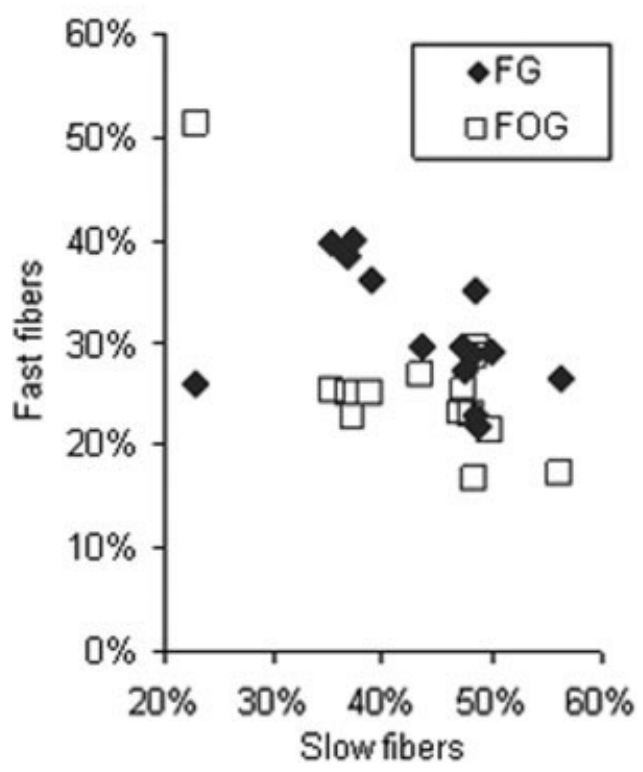

Fig. 1. Proportions of FG (dark diamonds) and FOG (open squares) versus SO fibers. 


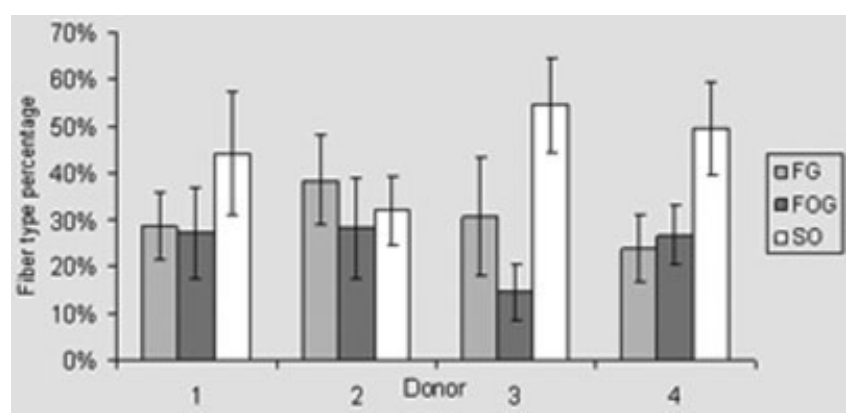

Fig. 2. Mean fiber type composition by donor. The respective ages for the donors 1-4 were as follows: 57, 51,75 , and 17 .

ence in fiber type proportions, except for the proportions of FOG fibers between abductors and adductors $(P=0.026)$.

Measurements of fiber type CSA demonstrated considerable variability among different muscles (Table 2). Additionally, there was considerable variability for the fiber type composition and CSA ratios by specimen. SO fibers were the most predominant fiber type for donors 1,3 , and 4 (respective ages of 57, 75, and 17 years-old). For donor 2, age 51, FG fibers contributed most to the fiber type composition. FOG fibers made the smallest contribution for donors 1,2 , and 3 . For donor 4, FG fibers contributed the least to the fiber type composition (Fig. 2). For donors 1 and 2, with respective ages of 57 and 51 years-old, the average CSA contribution for the FG fibers was less than that made by both the SO and FOG. For donor 3, age 75 years-old, the average FG fiber CSA was greater than the average FOG CSA and the average SO CSA. However, for donor 4, age 17 years-old, the average FG CSA was greater than the average SO CSA and less than the average FOG CSA (Fig. 3).

The maximum shortening velocities determined for each of the fourteen muscles ranged from 1.2 to $1.8 \mathrm{~L}_{0} / \mathrm{sec}$ (Table 2). Arranged from slowest to fastest, the order of muscles is as follows: anterior deltoid, teres minor, and teres major $\left(1.2 \mathrm{~L}_{0} / \mathrm{sec}\right)$, coracobrachialis and infraspinatus (1.3 $\left.\mathrm{L}_{0} / \mathrm{sec}\right)$, posterior deltoid and supraspinatus $\left(1.4 \mathrm{~L}_{0} /\right.$ $\mathrm{sec})$, triceps $(1.5 \mathrm{~L} 0 / \mathrm{sec})$, middle deltoid, latissimus dorsi, and subscapularis $(1.6 \mathrm{Lo} / \mathrm{sec})$, short head of biceps (1.7 $\left.\mathrm{L}_{0} / \mathrm{sec}\right)$, long head of biceps, and pectoralis $\left(1.8 \mathrm{~L}_{0} / \mathrm{sec}\right)$.

\section{DISCUSSION}

Fiber type data has been collected for muscles crossing the glenohumeral joint and has been used to estimate the maximum velocity of shortening of each muscle $\left(\mathrm{V}_{\max }\right)$; this is a crucial parameter when incorporating the force-velocity relationship into a musculoskeletal model. The data presented here increases our understanding of the performance of shoulder muscles under nonisometric conditions, and allows for models to be constructed for predicting muscle forces under conditions of muscle shortening or lengthening.

Little attention is given to values for $V_{\max }$ in upper extremity musculoskeletal models, despite the fact that maximum velocities of shortening for each muscle fiber type are relatively well established. The models are used to make muscle force predictions for isometric, isokinetic, and isotonic conditions. Prediction of muscle forces for the isomet- ric case is relatively simple. But in cases where the models are used to predict forces for conditions of muscle lengthening or shortening, predicted forces are dependent on the maximum velocity of shortening. The development of an upper extremity model for isokinetic conditions at increasing joint velocities, as has been done for other extremities, necessitates an accurate and complete dataset of muscle fiber type composition as well as maximum shortening velocities. This requirement is increasingly important when considering the current paradigm of measuring joint kinematics and calculating muscle kinematics (i.e., velocity of shortening at a point in time) for concentric and eccentric muscle conditions.

Models of the lower extremity have been used to make predictions of how various factors alter the $\mathrm{V}_{\max }$ and commensurate force generation in aged human subjects (Thelen et al., 2003). For the shoulder, such an examination would be interesting, but without a complete dataset, the exercise would be little more than a sensitivity analysis. The data presented here serves as a baseline set of values.

Of note, Johnson et al. (1973) measured muscle fiber types in six upper extremities for deltoid, triceps, supraspinatus, pectoralis major, latisimus dorsi, and biceps brachii but did not differentiate between FG and FOG fibers. When we examine the data from this study, combining FOG and FG fibers as fast fibers, we see relatively favorable comparison with that presented by Johnson et al. (1973) (Fig. 4).

Models of the shoulder and upper extremity have used a wide range of maximum shortening velocities. There appears to be no consensus of appropriate values. Zajac (1989) suggests that $10 \mathrm{~L}_{0} / \mathrm{sec}$ is appropriate, and this value was used in an early upper extremity version of SIMM (Delp and Loan, 1995). Other models have used $5 \mathrm{~L}_{0} / \mathrm{sec}$ (Happee and Van der Helm, 1995), and $10 \mathrm{~L} / \mathrm{sec}$ (Soechting and Flanders, 1997). The data presented here suggests that the maximum velocities of shortening of upper extremity muscles are less than the range of values used in these models. Consequently, the forces predicted from models using the values presented here would be greater than the values previously reported.

Practical neuromusculoskeletal models, whether they be based on optimal control (Pandy et al., 1995; Thelen et al.,

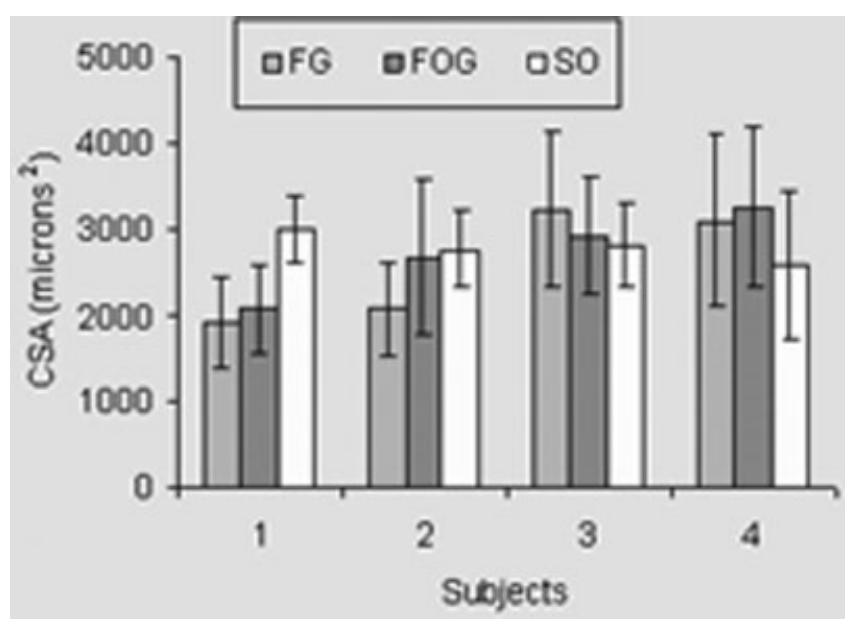

Fig. 3. Mean fiber CSA $\left(\mu \mathrm{m}^{2}\right)$ for each individual donor. The respective ages for the donors $1-4$ were as follows: $57,51,75$, and 17 . 


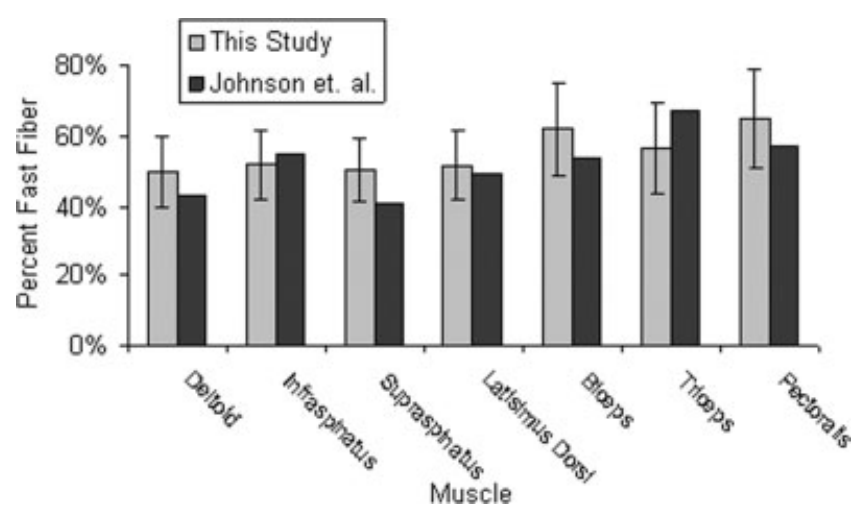

Fig. 4. Mean fast fiber contribution across donors for this study and for the study of Johnson et al. (1973). Johnson et al. (1973) reported deltoid and triceps measured at the surface and deep regions, and pectoralis major was measured for the sternal and clavicular heads. For these muscles, the values presented in this chart are the averages of values reported in Johnson et al. (1973).

2003) or filtered electromyographic signals (Manal et al., 2002; Langenderfer et al., 2005), assume an independence between neural activation and maximum shortening velocity. Some models assume linear scaling (Manal et al., 2002; Langenderfer et al., 2005). The size principle of motorunit recruitment, however, requires small motor units containing So fibers to be recruited first. That is, there is not a fixed proportion of fiber types recruited across activation level. Therefore, submaximal shortening velocity of a muscle may not scale linearly with activation. The maximum velocity of shortening data reported here should be used in modeling studies with this caveat in mind.

Previous studies have shown that fiber type composition and CSA varies with age (Klitgaard et al., 1990; Larsson et al., 1997). Because of the small number of donors used in this study, it was not possible to draw definitive conclusions regarding differences in fiber type composition and CSA relative to age. However, this data was collected on predominantly aged donors, and is therefore most appropriate for modeling muscle force for people in the fifth through seventh decades of life. Because of the tissue procurement method, a second limitation of this study was the inability to analyze fiber type distribution within different depths of the same muscle.

A third limitation of this study is that fiber type shortening velocities were not measured directly, but were taken from the literature (Larsson and Moss, 1993), and used to estimate muscle shortening velocities based on their proportion of CSA. Larsson and Moss (1993) reported shortening velocities for fibers obtained using two different methods: chemical skinning and freeze-drying. The mean maximum shortening velocities determined with the chemically skinned method were used in this study. While the results for type I fibers were similar using the two methods $(0.35$ \pm 0.16 vs. $0.31 \pm 0.14 \mathrm{~L}_{0} / \mathrm{sec}$ ), the velocities for the faster fibers were reduced for the freeze-drying method as compared to the chemically skinned results $(1.07 \pm 0.37 \mathrm{vs}$. $0.77 \pm 0.47$ for type IIA fibers, and 3.68 vs. $3.04 \pm 0.86$ for type IIB fibers). As a result, the data reported here may slightly overestimate maximum velocities of shortening, particularly for muscles composed of greater proportions of fast muscle fibers.

Despite its limitations, to date, this study is the most comprehensive description of muscle fiber type and maximum shortening velocities for muscles crossing the shoulder joint. The data presented here provide values for maximum velocities of shortening for use in musculoskeletal models, which can be used to predict physiologically based muscle forces for functionally relevant tasks under conditions of muscle shortening and lengthening.

\section{ACKNOWLEDGMENTS}

We would like to express appreciation to Gift of Life of Michigan for their assistance in obtaining muscle biopsies, to John Baker for his help with the staining protocol, Jan Hall for her help with the statistical analysis, and to Brian Childress for his contribution to data analysis.

\section{REFERENCES}

Bamman MM, Clarke MSF, Feeback DL, Talmadge RJ, Stevens BR, Lieberman SA, Greenisen MC. 1998. Impact of resistance exercise during bed rest on skeletal muscle sarcopenia and myosin isoform distribution. J Appl Physiol 84:157-163.

Bárány M. 1967. ATPase activity of myosin correlated with speed of muscle shortening. J Gen Physiol 50(Suppl):197-218.

Burke RE. 1981. Motor Units: Anatomy, Physiology, and Functional Organization. In: Brookhart JM, Mountcastle VB, Brooks VB, Geiger SR, editors. Handbook of Physiology. Bethesda, MD: American Physiological Society. p 345-422.

Cheng EJ, Brown IE, Loeb GE. 2000. Virtual muscle: A computational approach to understanding the effects of muscle properties on motor control. J Neurosci Methods 101:117-130.

Delp SL, Loan JP. 1995. A graphics-based software system to develop and analyze models of musculoskeletal structures. Comput Biol Med 25:21-34.

Fitts RH, Costill DL, Gardetto PR. 1989. Effect of swim exercise training on human muscle fiber function. J Appl Physiol 66:465-475.

Gansen H-K, Irlenbusch U. 2002. Neuromuscular failure of the rotator cuff as a contribution to the functional impingement of the shoulder-A muscle biopsy investigation. Z Orthop Ihre Grenzgeb 140:65-71.

Guth L, Samaha FJ. 1970. Procedure for the histochemical demonstration of actomyosin ATPase. Exp Neurol 28:365-367.

Happee R, Van der Helm FCT. 1995. The control of shoulder muscles during goal directed movements, an inverse dynamic analysis. J Biomech 28:1179-1191.

Hatze H. 1978. A general myocybernetic control model of skeletal muscle. Biol Cybern 28:143-157.

Hatze H. 1981. Estimation of myodynamic parameter values from observations on isometrically contracting muscle groups. Eur J Appl Physiol Occup Physiol 46:325-338.

Jennekens FGI, Tomlinson BE, Walton JN. 1971. Data on the distribution of fibre types in five human limb muscles. J Neurol Sci 14:245-257.

Johnson MA, Polgar J, Weightman D, Appleton D. 1973. Data on the distribution of fibre types in thirty-six human muscles. An autopsy study. J Neurol Sci 18:111-129.

Jozsa L, Demel Z, Vandor E, Reffy A, Szilagyi I. 1978. Specific fibre composition of human hand and arm muscles. Handchirurgie $10: 153-157$.

Jürimäe J, Abernethy PJ, Blake K, McEniery MT. 1996. Changes in the myosin heavy chain isoform profile of the triceps brachii muscle following 12 weeks of resistance training. Eur J Appl Physiol Occup Physiol 74:287-292.

Kaufman KR, An KN, Litchy WJ, Chao EYS. 1991. Physiological prediction of muscle forces. I. Theoretical formulation. Neuroscience 40:781-792. 
Klitgaard H, Zhou M, Schiaffino S, Betto R, Salviaiti G, Saltin B. 1990. Ageing alters the myosin heavy chain composition of single fibers from human skeletal muscle. Acta Physiol Scand 140:55-62.

Langenderfer J, LaScalza S, Mell A, Carpenter JE, Kuhn JE, Hughes RE. 2005. An EMG-driven model of the upper extremity and estimation of long head biceps force. Comput Biol Med 35:25-39.

Larsson L, Moss RL. 1993. Maximum velocity of shortening in relation to myosin isoform composition in single fibres from human skeletal muscles. J Physiol 472:595-614.

Larsson L, Li X, Frontera WR. 1997. Effects of aging on shortening velocity and myosin isoform composition in single human skeletal muscle cells. Am J Physiol 272:C638-C649.

Manal K, Gonzalez RV, Lloyd DG, Buchanan TS. 2002. A real-time EMG-driven virtual arm. Comput Biol Med 32:25-36.

McGill SM, Norman RW. 1986. Partitioning of the L4-L5 dynamic moment into disc, ligamentous, and muscular components during lifting. Spine 11:666-678.

McIntosh JS, Ringqvist M, Schmidt EM. 1985. Fiber type composition of monkey forearm muscle. Anat Rec 211:403-409.

Pandy MG, Garner BA, Anderson FC. 1995. Optimal control of nonballistic muscular movements: A constraint-based performance criterion for rising from a chair. J Biomech Eng 117:15-25.

Portney LG, Watkins MP. 1993. Foundations of Clinical Research: Applications to Practice. Norwalk, CT: Appleton and Lang.

Richmond FJR, Singh K, Corneil BD. 1999. Marked non-uniformity of fiber-type composition in the primate suboccipital muscle obliquus capitis inferior. Exp Brain Res 125:14-18.

Scott SH, Brown IE, Loeb GE. 1996. Mechanics of feline soleus. I. Effect of fascicle length and velocity on force output. J Muscle Res Cell Motil 17:207-219.
Scott W, Stevens J, Binder-Macleod SA. 2001. Human skeletal muscle fiber type classifications. Phys Ther 81:1810-1816.

Singh K, Melis EH, Richmond FJR, Scott SH. 2002. Morphometry of Macaca mulatta forelimb. II. Fiber-type composition in shoulder and elbow muscles. J Morphol 251:323-332.

Smerdu V, Karsch-Mizrachi I, Campione M, Leinwand L, Schiaffino S. 1994. Type IIx myosin heavy chain transcripts are expressed in type IIb fibers of human skeletal muscle. Am J Physiol 267: C1723-C1728.

Soechting JF, Flanders M. 1997. Evaluating an integrated musculoskeletal model of the human arm. J Biomech Eng 119:93102.

Spector SA, Gardiner PF, Zernicke RF, Roy RR, Edgerton VR. 1980 Muscle architecture and force-velocity characteristics of cat soleus and medial gastrocnemius: Implications for motor control. ] Neurophysiol 44:951-960.

Thelen DG. 2003. Adjustment of muscle mechanics model parameters to simulate dynamic contractions in older adults. J Biomech Eng 125:70-77.

Thelen DG, Anderson FC, Delp SL. 2003. Generating dynamic simulations of movement using computed muscle control. J Biomech 36:321-328.

Thorstensson A, Grimby G, Karlsson J. 1976. Force-velocity relations and fiber composition in human knee extensor muscles. J Appl Physiol 40:12-16.

Trappe S, Costill D, Thomas R. 2000. Effect of swim taper on whole muscle and single fiber contractile properties. Med Sci Sports Exerc 33:48-56.

Zajac FE. 1989. Muscle and tendon: Properties, models, scaling, and application to biomechanics and motor control. Crit Rev Biomed Eng 17:359-411. 